\title{
GABAergic Circuits Control Input-Spike Coupling in the Piriform Cortex
}

\author{
Victor M. Luna and Nathan E. Schoppa \\ Department of Physiology and Biophysics, University of Colorado at Denver, Anschutz Medical Campus, Aurora, Colorado 80045
}

Odor coding in mammals is widely believed to involve synchronized gamma frequency $(30-70 \mathrm{~Hz})$ oscillations in the first processing structure, the olfactory bulb. How such inputs are read in downstream cortical structures however is not known. Here we used patchclamp recordings in rat piriform cortex slices to examine cellular mechanisms that shape how the cortex integrates inputs from bulb mitral cells. Electrical stimulation of mitral cell axons in the lateral olfactory tract (LOT) resulted in excitation of pyramidal cells (PCs), which was followed $\sim 10 \mathrm{~ms}$ later by inhibition that was highly reproducible between trials in its onset time. This inhibition was somatic in origin and appeared to be driven through a feedforward mechanism, wherein GABAergic interneurons were directly excited by mitral cell axons. The precise inhibition affected action potential firing in PCs in two distinct ways. First, by abruptly terminating PC excitation, it limited the PC response to each EPSP to exactly one, precisely timed action potential. In addition, inhibition limited the summation of EPSPs across time, such that PCs fired action potentials in strong preference for synchronized inputs arriving in a time window of $<5$ ms. Both mechanisms would help ensure that PCs respond faithfully and selectively to mitral cell inputs arriving as a synchronized gamma frequency pattern.

Key words: olfactory; piriform cortex; synchronization; GABA; mitral cell; inhibition

\section{Introduction}

A prominent feature of the mammalian olfactory system is synchronization of neuronal activity. Synchrony has been best characterized in the first processing structure, the olfactory bulb, where it appears in the form of both local field potential (LFP) oscillations at the gamma frequency $(30-70 \mathrm{~Hz}$; Adrian, 1950; Freeman, 1972), as well as in action potential firing ("spiking") in the output neurons of the bulb, the mitral cells (Kashiwadani et al., 1999; Schoppa, 2006). Functionally, synchrony in the bulb has been widely proposed to facilitate the integration of information about different odorant receptors (ORs) that are activated by an odor (Mori et al., 1999; Lledo et al., 2005). Indeed, it has been shown that odor discrimination is impaired when synchrony is pharmacologically disrupted in the insect antennal lobe, the structure analogous to the mammalian bulb (Stopfer et al., 1997). What remains unclear however is exactly how synchrony in the bulb is read by downstream regions involved in olfactory processing, specifically pyramidal cells (PCs) in the piriform cortex, the structure that receives most of the direct synaptic inputs from mitral cells (Neville and Haberly, 2004). In particular, if synchrony is important functionally, might there be mechanisms in the piriform cortex that make PCs prefer synchronized signals from mitral cells?

\footnotetext{
Received May 23, 2008; revised July 10, 2008; accepted July 31, 2008.

This work was supported by National Institutes of Health Grant R01 DC006640. We thank the Light Microscopy Facility at University of Colorado at Denver for assistance with imaging.

Correspondence should be addressed to Nathan E. Schoppa, Department of Physiology and Biophysics, University of Colorado at Denver, Anschutz Medical Campus, Mail Stop 8307, P.0. Box 6511, Aurora, C0 80045. E-mail: nathan.schoppa@uchsc.edu.

D0I:10.1523/JNEUROSCI.2385-08.2008

Copyright $\odot 2008$ Society for Neuroscience $\quad 0270-6474 / 08 / 288851-09 \$ 15.00 / 0$
}

The piriform cortex has a number of synaptic mechanisms that could influence how PCs integrate incoming glutamatergic signals from mitral cells. For example, PCs receive extensive recurrent excitatory connections from other PCs in the piriform cortex, as well as from outside cortical areas (Neville and Haberly, 2004). Field potential recordings in slices have shown that these synapses can introduce a delayed, polysynaptic excitatory response component after stimulation of mitral cell axons (Rodriguez and Haberly, 1989; Ketchum and Haberly, 1993), which could influence PC responses under conditions of repeated stimulation. PCs also receive inputs from GABAergic interneurons that can drive both $\mathrm{GABA}_{\mathrm{A}}$ and $\mathrm{GABA}_{\mathrm{B}}$ receptor-mediated synaptic potentials (Biedenbach and Stevens, 1969; Tseng and Haberly, 1988; Kapur et al., 1997). Depending on the polarity and timing of these potentials, GABAergic inputs could also have potent effects on PC spike responses (Pouille and Scanziani, 2001).

In this study, we performed patch-clamp recordings in slices of rat anterior piriform cortex to examine how its intrinsic circuitry impacts the way PCs integrate synaptic inputs from mitral cells. When stimulating mitral cell axons in the lateral olfactory tract (LOT), PC responses included a fast $\mathrm{GABA}_{\mathrm{A}}$ receptormediated synaptic component that occurred with a precise onset time regardless of the strength of local circuit activation. These GABAergic events appeared to be somatic in origin, were likely generated through a feedforward mechanism, and contributed a larger fraction of the total synaptic response under conditions of high frequency stimulation that mimicked natural stimuli. In functional studies of PC spike activity, we found that the GABAergic events had an inhibitory action on PC responses to 
single bouts of mitral cell input and also affected how PCs integrated multiple mitral cell inputs across time.

\section{Materials and Methods}

Parasagittal slices $(350 \mu \mathrm{m})$ were prepared from the anterior piriform cortices of 9- to 14-d-old Sprague Dawley rats by using procedures modified from those previously described for preparation of olfactory bulb slices (Schoppa et al., 1998). Slices were visualized with a $40 \times$ waterimmersion objective on an Axioskop 2 FS plus microscope (Carl Zeiss) using differential interference contrast (DIC) optics. The anterior piriform cortex was identified based on location relative to the LOT and compact density of layer 2 cells. Experiments were done at $30-33^{\circ} \mathrm{C}$. All experiments were approved by the Institutional Animal Care and Use Committee at University of Colorado at Denver (UCD).

The base extracellular solution in all experiments contained (in $\mathrm{mm}$ ): $125 \mathrm{NaCl}, 25 \mathrm{NaHCO}_{3}, 1.25 \mathrm{NaH}_{2} \mathrm{PO}_{4}$, 25 glucose, $2.5 \mathrm{KCl}, 1 \mathrm{MgCl}_{2}, 2$ $\mathrm{CaCl}_{2}, \mathrm{pH}$ 7.3. Except where noted, whole-cell recordings were done with pipette solutions containing (in $\mathrm{mm}$ ): $150 \mathrm{~K}$-gluconate, 5 HEPES, 1.1 EGTA, $1 \mathrm{MgCl}_{2}, 10$ phosphocreatine, $\mathrm{pH}$ 7.2. In some recordings, the pipette solution included $0.2 \%$ biocytin for subsequent examination of morphology. For these cells, slices were fixed in a $4 \%$ formaldehyde solution, exposed to Cy-5-conjugated streptavidin $(1 \mu \mathrm{g} / \mathrm{ml}$; Jackson ImmunoResearch), and visualized on a confocal microscope (Zeiss LSM 510 NLO; Light Microscopy Facility at UCD). For loose cell-attached patch recordings, pipettes were filled with the extracellular solution.

Identification of cell types was based on the following criteria. Layer 2 PCs, the main focus of these studies, were located in deep layer 2 of the piriform cortex, were relatively large in size ( $\sim 20 \mu \mathrm{m}$ diameter $)$, and had a triangular/apical-taper shape. Of 15 putative PCs cells in layer 2 in which detailed morphology was examined using biocytin fills, 13 clearly had the previously described morphological characteristics of layer 2 PCs (Neville and Haberly, 2004; Suzuki and Bekkers, 2006), with long, extensively branched apical dendrites and shorter but well branched basal dendrites (see Fig. 1A). Layer 3 PCs were identified based on location (layer 3$)$, their large size $(\sim 25 \mu \mathrm{m}$ diameter), and shape (triangular/ apical taper). GABAergic interneurons were identified based on their location (near the border of layer $1 \mathrm{~b}$ and superficial layer 2), their smaller size ( $\sim 10 \mu \mathrm{m}$ diameter), and oblong or teardrop shape. Three putative interneurons in which more detailed morphology was examined using biocytin fills had the characteristics of vasoactive intestinal peptide (VIP)/cholecystokinin (CCK)-expressing interneurons described by Ekstrand and coworkers (2001), including apical dendrites with short stems before branching and sparse basal dendrites. The mean resting potentials were $-69 \pm 2 \mathrm{mV}$ for layer $2 \mathrm{PCs}(n=13)$ and $-62 \pm 3 \mathrm{mV}$ for interneurons $(n=15)$. PCs with cut apical dendrites in Figure 2 had a mean resting potential of $-69 \pm 2 \mathrm{mV}(n=3)$.

Unless otherwise indicated, electrical stimulation was done using a broken-tip patch pipette $(4-6 \mu \mathrm{m})$ positioned directly on the LOT. Single stimulus pulses or stimulus patterns were generated by the computer, which then triggered a stimulus isolator (SYS-A365D; World Precision Instruments). Stimulus protocols were delivered every 5-10 s. Current and voltage signals were recorded with a MultiClamp 700A dual patch clamp (Axon Instruments), digitized at $5-10 \mathrm{kHz}$, and filtered at $2.5-4$ $\mathrm{kHz}$. Unless otherwise stated, PCs were held at -47 to $-62 \mathrm{mV}$ during voltage-clamp recordings to allow visualization of both excitatory and inhibitory components of the synaptic response (as inward and outward currents, respectively). In current-clamp recordings of PC spiking (see Figs. $5 B, 6$ ), positive current was typically injected to depolarize cells somewhat (generally between -40 and $-45 \mathrm{mV}$ ) before stimulation. Data were acquired and analyzed on a Macintosh G5 computer using Axograph software.

Drug solutions were bath-applied in most experiments. For the "puffer" experiments in Figure 2, bicuculline methiodide (BMI) and 2,3,dihydroxy-6-nitro-7-sulfamoyl-benzo-f-quinoxaline (NBQX) were applied through a patch pipette (1-2 $\mu \mathrm{m}$ diameter) using a Picospritzer III (Parker Hannifin Instrumentation). The puffer solution also included $1 \%$ phenol red. Each puff was applied at $2-5$ psi, which limited the phenol red signal to a localized area in either layer la or layer 2 of the piriform cortex. Each puff began $500 \mathrm{~ms}$ before LOT stimulation and lasted $500 \mathrm{~ms}$.

Data values are reported as mean \pm SE. Student's $t$ test was used to determine statistical significance ( $p<0.05$; indicated by single asterisks in figures).

\section{Results}

Fast, precisely timed inhibition onto pyramidal cells

To examine the dynamics of the piriform cortex circuitry, we measured voltage-clamp responses in PCs to single shocks (100 $\mu \mathrm{s}$ ) applied to the LOT (holding potential, -47 to $-62 \mathrm{mV}$ ) (Fig. $1 A)$. We focused on PCs located in layer 2 of the structure (see diagram in Fig. $1 A$ ), which in the anterior piriform cortex are at a higher density than in layer 3 (Neville and Haberly, 2004). The exact nature of the PC response was dependent on the stimulus intensity. At weak intensities $(<50 \mu \mathrm{A})$ (Fig. $1 \mathrm{~B})$, responses generally consisted of only a fast inward EPSC (amplitude, $-29 \pm 5$ pA; half-width, $11.3 \pm 0.8 \mathrm{~ms} ; n=10$ cells), reflecting monosynaptic transmission from LOT to PCs (Franks and Isaacson, 2006). At higher stimulus intensities ( $\geq 30 \mu \mathrm{A}$ ) (Fig. 1C,D), responses typically also included (in 37 of 40 cells tested) fast, outward synaptic current events (10-90\% rise time, $1.0 \pm 0.1 \mathrm{~ms}$; halfwidth, $4.7 \pm 0.5 \mathrm{~ms} ; n=5$ cells) that were blocked by the $\mathrm{GABA}_{\mathrm{A}}$ receptor antagonist BMI $(20 \mu \mathrm{M} ; n=6)$ (Fig. $1 D$, inset). These GABAergic events were also blocked by the AMPA/kainate receptor antagonist NBQX (10 $\mu \mathrm{M} ; n=6$; data not shown), indicating that they did not result from direct activation of GABAergic synapses and instead reflected secondary activation of local circuits by LOT inputs.

Figure 1, $C$ and $D$, separates GABAergic responses based on the strength of stimulation of the local circuitry, as defined by whether there were significant failures in eliciting GABAergic events ("perithreshold" conditions; failure rate of 33-76\% across seven cells; stimulus intensity, 30-100 $\mu \mathrm{A}$ ) or no failures ("suprathreshold"; stimulus intensity, 70-400 $\mu \mathrm{A}$ ). Responses under these two conditions differed markedly in the number of evoked GABAergic events $(1.2 \pm 0.2$ vs $4.4 \pm 0.5$ events per stimulus for peri- and suprathreshold stimuli, respectively; $n=7$ ), yet a consistent aspect was a relatively early event that occurred in $\sim 10 \mathrm{~ms}$, which differed only slightly in delay (Fig. $1 E$ ) (mean delay after stimulus, $14.5 \pm 1.5$ and $8.9 \pm 0.7 \mathrm{~ms}$ for perithreshold and suprathreshold stimuli, respectively; $n=7 ; p=0.02$ using paired $t$ test). This event had a $\sim 10 \mathrm{~ms}$ delay at even the highest stimulus intensities tested $(0.5-8 \mathrm{~mA} ; 8.5 \pm 1.0 \mathrm{~ms} ; n=4)$. Under all conditions, the GABAergic event occurred with remarkably little jitter between trials, typically varying by only $1-2 \mathrm{~ms}$ in onset time (SD in onset time per cell, $1.0 \pm 0.2 \mathrm{~ms}$ and $0.5 \pm 0.1 \mathrm{~ms}$ for peri- and suprathreshold stimuli, respectively; $n=7 ; p=0.10$ using paired $t$ test). Thus, it appears that at least part of the GABAergic local circuitry in the piriform cortex is configured to produce precise synaptic input onto PCs regardless of the strength of network activation. The fast event occurring at $\sim 10$ $\mathrm{ms}$ was also the most persistent component of the GABAergic response when stimulus trains were applied near the gamma frequency to mimic natural odor conditions (Fig. $1 F$ ). Ten pulses at $20-33 \mathrm{~Hz}$ (stimulus intensity, $400-600 \mu \mathrm{A} ; n=7$ ) resulted in outward-going synaptic responses that were consistently converted from a complex waveform that included both early and delayed components (as in Fig. 1D) to a single fast-occurring event that happened with a $\sim 10 \mathrm{~ms}$ delay. This change was reflected as a reduction in the duration of the average "composite" outward current response evoked by each stimulus (mean half- 

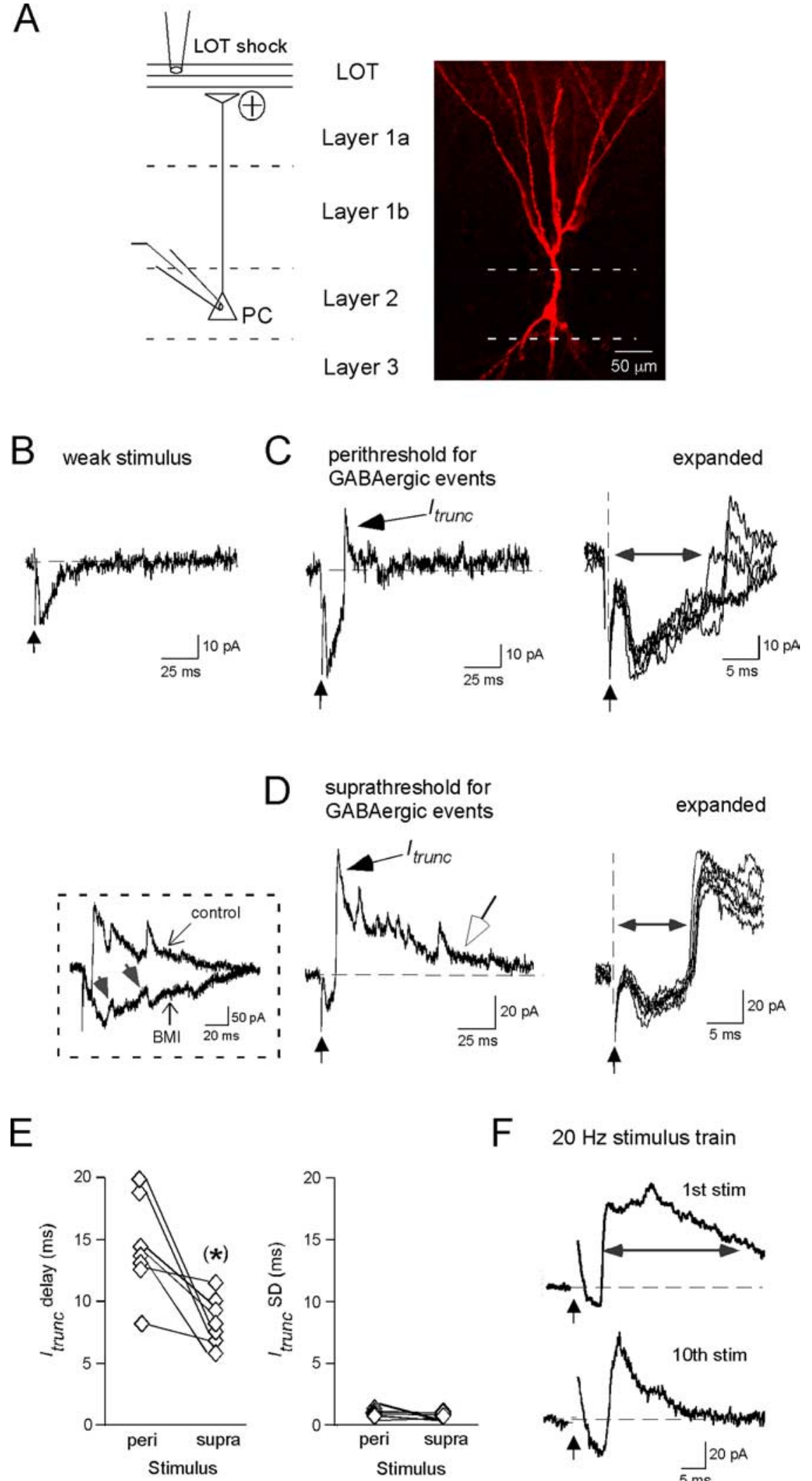

F $20 \mathrm{~Hz}$ stimulus train

Figure 1. GABAergic interneurons mediate highly reproducible synaptic events in PCS. $A$, Left, Diagram illustrating a PC juxtaposed against the different layers of the anterior piriform cortex. Most recordings were done on deep layer $2 \mathrm{PCs}$, a biocytinfilled example of which is shown on the right. $\boldsymbol{B}$, Monosynaptic EPSC evoked by a weak LOT shock (time indicated by vertical arrow). C, Left, A typical response at a higher stimulus intensity $(100 \mu \mathrm{A})$, showing a fast-rising GABAergic event (called $I_{\text {trunc }}$ ) that acted to truncate the monosynaptic EPSC. The displayed response was recorded under "perithreshold" conditions, i.e., when the failure rate for GABAergic events was significant ( $81 \%$ in this experiment). Right, Five superimposed expanded traces highlighting the reproducible delay to $I_{\text {trunc }}$. The delay time (horizontal double-arrowed line) was measured as the time from the stimulus artifact to the half-amplitude of $I_{\text {trunc }}$. , In other recordings at even higher stimulus intensities (in this case, $300 \mu \mathrm{A}$ ), GABAergic width of composite response, $25.4 \pm 4.8$ $\mathrm{ms}$ and $8.4 \pm 1.1 \mathrm{~ms}$, after the first and tenth stimuli, respectively; $n=7 ; p=$ 0.007).

In the rest of this study, we will focus on the component of the GABAergic response that occurred with a fast rise time and relatively short delay (in $\sim 10 \mathrm{~ms}$ ) after LOT stimulation, examining its mechanisms and function. We will refer to this current as $I_{\text {trunc }}$, based on the fact that it appeared to truncate the EPSC associated with LOTto-PC transmission (Fig. 1C,D).

Mechanisms generating $I_{\text {trunc }}$

Within other brain circuits (see, e.g., Miles et al., 1996), GABAergic inputs have been shown to be most effective at altering sodium channel-dependent spikes in target cells when inputs are localized near the cell soma. To assess the origin of $I_{\text {trunc }}$ in PCs in the piriform cortex, we tested for blockade of $I_{\text {trunc }}$ when BMI $(30 \mu \mathrm{M})$ was puffapplied onto various layers of the piriform slice (Fig. 2A-C). Application of BMI in layer 2, near the soma of the test PC, resulted in rapid reduction of $I_{\text {trunc }}(58 \pm 8 \%$ reduction in peak current after first puff; $n=7 ; p=0.031$ ), whereas application at the apical dendrite in layer 1a (25-50 $\mu \mathrm{m}$ below the edge of the LOT) yielded no effect ( $15 \pm 8 \%$ increase; $n=5)$. In the latter studies, we controlled for drug access to dendritic synaptic sites by showing, in parallel experiments, that NBQX $(5-10 \mu \mathrm{M})$ puff-applied in layer 1a reduced EPSCs resulting from LOT-to-PC transmission (29 $\pm 9 \%$ decrease after first puff; $n=8$; $p=0.025)$. The differential effect of BMI in layer 2 versus 1a implies that $I_{\text {trunc }}$ orig-

activity occurred as a barrage of multiple fast events. In such cases, the GABAergic events were seen in all trials ("suprathreshold"). The five superimposed expanded traces on the right show that, under these conditions, there also is an I trunc event that happens with very little jitter. The open arrow on the unexpanded trace indicates a slower outward synaptic current that appeared to be independent of the fast superimposed IPSCs. Left (boxed inset), Bath application of $20 \mu \mathrm{m}$ BMI eliminated the fast outward events, indicating that they were mediated by $G A B A_{A}$ receptors. Small upward deflections in the remaining response (gray arrows) do not have the fast rise times and exponential decays predicted for GABAergic events and instead likely reflect polysynaptic EPSCS. $\boldsymbol{E}$, Summary of $I_{\text {trunc }}$ delay and variability (SD) seen under perithreshold and suprathreshold conditions across seven cells. Statistically significant difference, ${ }^{*} p<0.05$. $\boldsymbol{F}, \mathrm{PC}$ responses measured during a $20 \mathrm{~Hz}$ stimulus train (10 LOT shocks at $500 \mu \mathrm{A})$. Averaged responses (20 trials) to the first and tenth stimuli are shown. Note the marked decrease in duration of the "composite" outward synaptic current (horizontal line demarcates half-width measurement), such that by the tenth stimulus, the GABAergic response was dominated by $I_{\text {trunc }}$. 


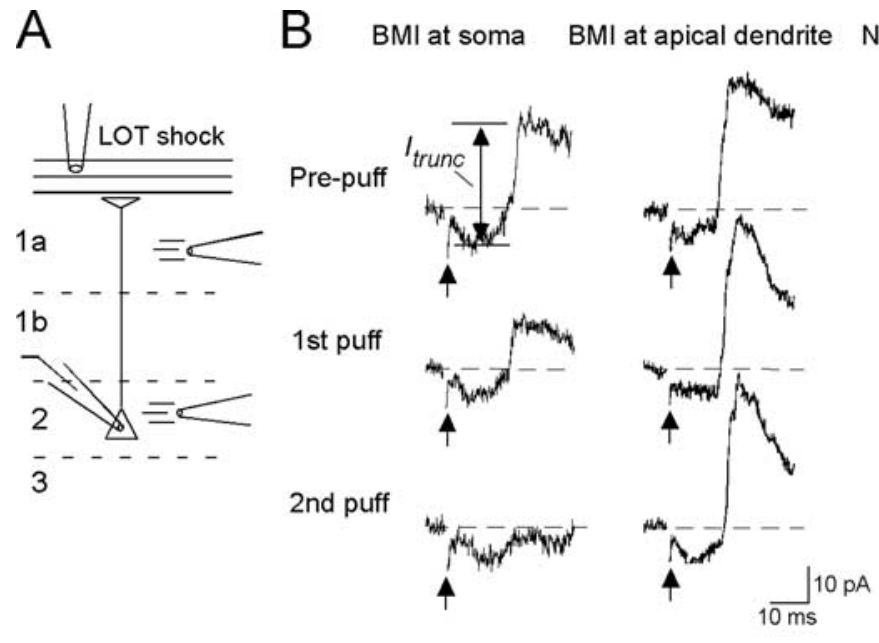
NBQX at apical dendrite $\mathrm{C}$
(weak stimulus)
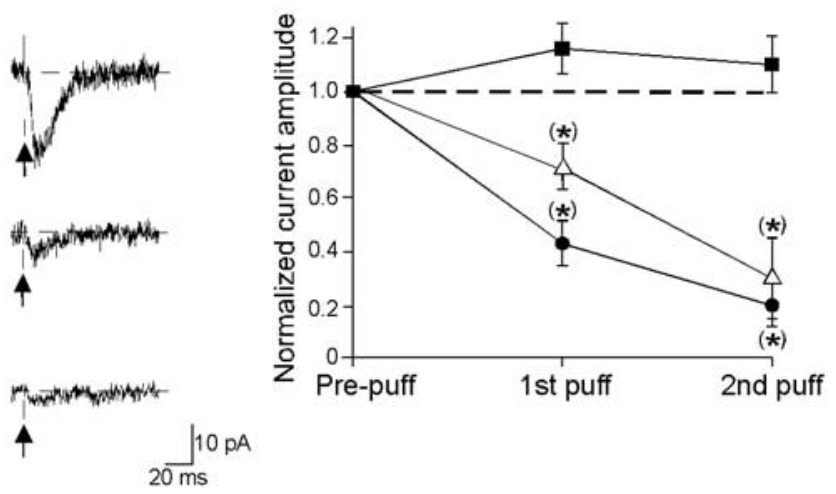

$\mathrm{D}$
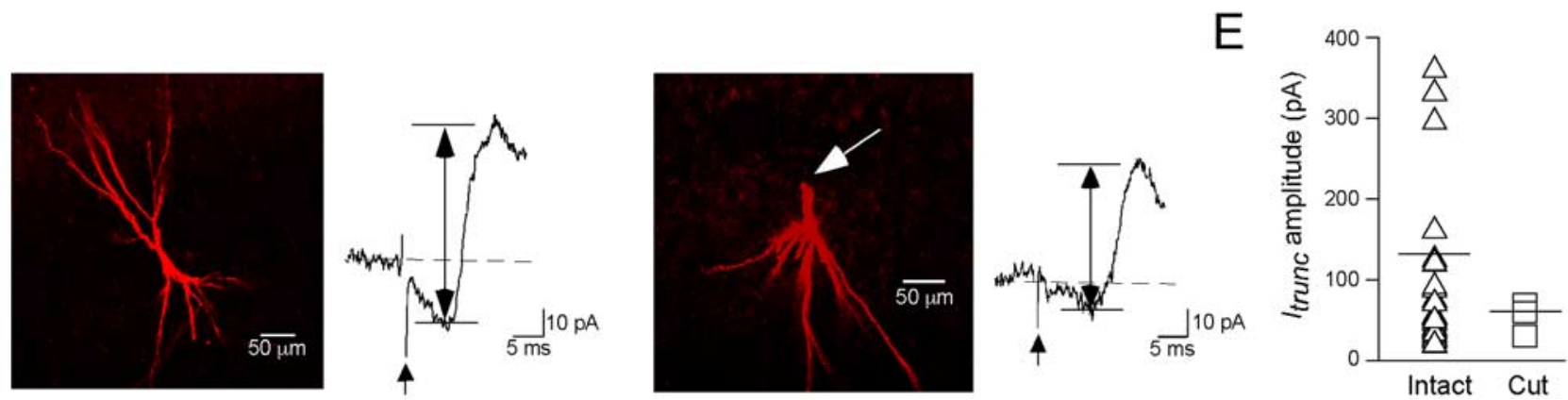

Figure 2. $I_{\text {trunc }}$ originates near the PC soma. $A$, Schematic of the puff application procedure used to localize $I_{\text {trunc }}$. The LOT was given a single shock ( $\left.300 \mu A\right)$ while a patch pipette was used to apply BMI or NBQX onto layer 1a or layer 2 to target the PC apical dendrite or soma, respectively. B, Sample traces of / trunc evoked before (prepuff) and after 1 or 2 puffs of $30 \mu \mathrm{m}$ BMI at the soma (left traces)

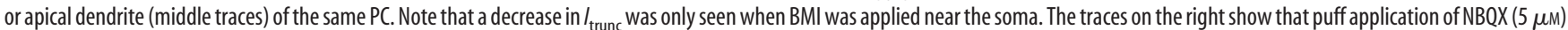
at the apical dendrite reduced the monosynaptic EPSC induced by weak LOT stimulation, indicating good drug access. All traces are single trials. The upward-pointing arrows indicate time of LOT stimulation; drug application started $500 \mathrm{~ms}$ before each stimulus. C, Summary of puffer experiments, showing that BMl application near the soma, but not the apical dendrite, consistently reduced $I_{\text {trunc }}$ compared with prepuff conditions. Also shown are results with NBQX application at apical dendrites. $D$, Two biocytin-filled cells, one with a fully intact apical dendrite (left) and another with

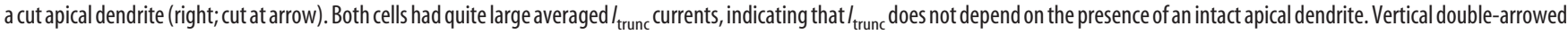
lines indicate estimated amplitudes of $I_{\text {trunc }} \boldsymbol{E}$, Summary of results comparing $I_{\text {trunc }}$ amplitude in PCs with intact or cut apical dendrites. The mean $I_{\text {trunc }}$ amplitudes for the two groups (horizontal lines) were not significantly different.

inated near the PC soma. Also consistent with this interpretation, we found that direct electrical stimulation of inputs in layer 2 (done in the presence of kynurenate, $2 \mathrm{~mm}$, to block glutamatergic transmission) elicited fast-rising GABAergic events (10-90\% rise time, $1.4 \pm 0.2 \mathrm{~ms}$, time to half-peak, $2.2 \pm 0.3 \mathrm{~ms} ; n=6$; data not shown), characteristic of $I_{\text {trunc }}$, but stimulation in layers $1 \mathrm{a}$ or b resulted in slower events (10-90\% rise time, $3.2 \pm 0.6 \mathrm{~ms}$; $n=9$ cells; $p=0.015)$. A last argument that $I_{\text {trunc }}$ originated near the PC soma was anatomical (Fig. $2 D, E$ ). In experiments in which we assessed the morphology of test PCs with biocytin fills, there was no relationship between the size of $I_{\text {trunc }}$ and whether test PCs had intact or sectioned apical dendrites (peak $I_{\text {trunc }}=$ $139 \pm 33 \mathrm{pA} ; n=12$, and $63 \pm 15 \mathrm{pA} ; n=3$, for intact vs sectioned cells, respectively; $p=0.30)$. $I_{\text {trunc }}$ in the sectioned cells also had typical $\sim 10 \mathrm{~ms}$ onset delays $(10.5 \pm 1.7 \mathrm{~ms} ; n=3)$. In the sectioned cells, no cell had an apical dendrite longer than 40 $\mu \mathrm{m}$, indicating that many of the input sites contributing to $I_{\text {trunc }}$ were within $40 \mu \mathrm{m}$ of the PC soma.

A second mechanistic issue pertains to the architecture of the GABAergic local circuit that yielded $I_{\text {trunc }}$. Based on the anatomy of the piriform cortex (Neville and Haberly, 2004), $I_{\text {trunc }}$ could originate through two circuits (Fig. $3 A$ ): a "feedforward" mechanism, wherein the GABAergic interneurons that produce $I_{\text {trunc }}$ in the test PC are activated directly by LOT inputs, or a "lateral" mechanism, where interneurons are activated secondarily by other PCs that are first activated by the LOT. To distinguish between these mechanisms, we first compared the stimulus intensity range over which $I_{\text {trunc }}$ was seen in PCs with when PCs spiked, the latter being monitored with loose cell-attached recordings. A lateral, but not feedforward, mechanism depends on PCs being activated before $I_{\text {trunc }}$, and thus $I_{\text {trunc }}$ should not be seen until stimulus intensities are reached that activate PCs. Figure 3, $B$ and $C$, shows the results of this analysis, done under perithreshold conditions for evoking spikes or $I_{\text {trunc }}$ (spike failure rate, $32-80 \%$; $I_{\text {trunc }}$ failure rate, $33-81 \%$ ) in a PC. In fact, the distributions of perithreshold stimuli for spikes in PCs and $I_{\text {trunc }}$ showed a significant mismatch. In all recordings of PC spiking, spikes only happened at stimulus intensities that were above, generally well above, that required to elicit $I_{\text {trunc }}$. Similar spike properties were seen for PCs in layer 2 (perithreshold intensities $\geq 260 \mu \mathrm{A}$ in nine cells) and layer 3 (perithreshold intensities $\geq 180 \mu \mathrm{A}$ in five cells). Although we could not completely exclude the possibility of a very small population of highly sensitive PCs missed in our experiments that could mediate a lateral mechanism, these results are most consistent with a feedforward mechanism for $I_{\text {trunc }}$. A similar conclusion was reached from the distributions of spike versus $I_{\text {trunc }}$ delay times in PCs for the same set of perithreshold experiments (Fig. 3D). In this case, the distributions were similar, with indistinguishable means $[12.8 \pm 1.9 \mathrm{~ms}$ $(n=14)$ and $14.5 \pm 1.5 \mathrm{~ms}(n=7)$ for PC spikes and $I_{\text {trunc }}$ delays, 
A

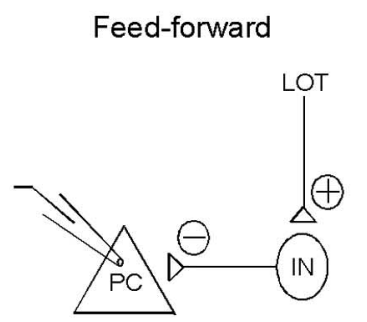

Lateral

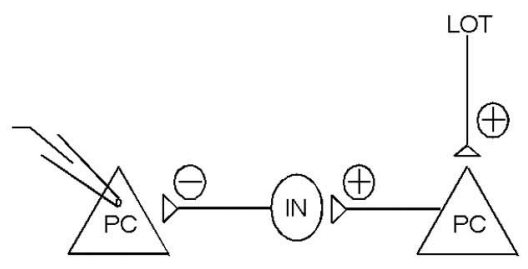

B

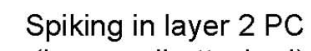
(loose cell-attached)

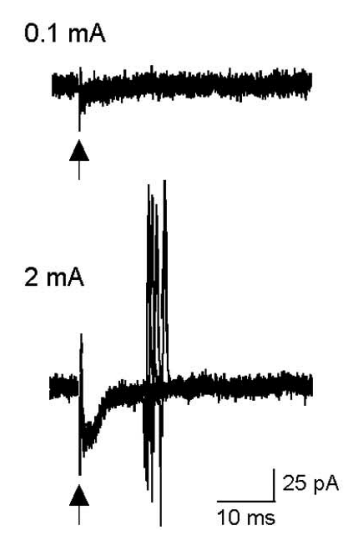

\section{C}

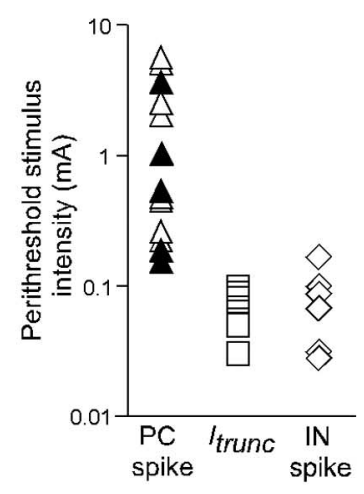

$\mathrm{D}$

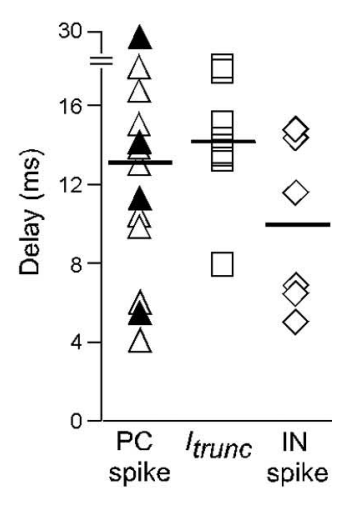

Figure 3. Evidence that $I_{\text {trunc }}$ arises through a feedforward mechanism. $A$, Two possible circuits responsible for $I_{\text {trunc }}$ a feedforward mechanism (left), in which the GABAergic interneuron (IN) that drives $I_{\text {trunc }}$ is directly excited by the LOT, and a lateral mechanism (right), in which the interneuron is excited by a multisynaptic path involving the LOT and another PC. B, Loose cell-attached recordings of PC activity at moderate (100 $\mu \mathrm{A}$; top) and high ( $2 \mathrm{~mA}$; bottom) stimulus intensities. Each panel shows 10 superimposed traces. Note that spikes were seen only at high intensities in 5 of the 10 trials. C, Scatter-plot generated from multiple PC recordings comparing the stimulus intensities that were perithreshold for evoking spikes (triangles) or $I_{\text {trunc }}$ (squares). Note that $I_{\text {trunc }}$ was observed at much lower stimulus intensities $(0.03-0.1 \mathrm{~mA})$ than $\mathrm{PC}$ spikes $(0.18-7 \mathrm{~mA})$. This mismatch is consistent with a feedforward mechanism for $I_{\text {trunc }}$. $O$ pen and filled triangles reflect $P C$ in layers 2 and 3 , respectively. Also plotted at right (diamonds) for comparison are perithreshold stimulus intensities for spiking in GABAergic interneurons in piriform cortex (IN; see Fig. 4). D, Scatter-plot comparing the delay times for spikes and $I_{\text {trunc }}$ in PCs under perithreshold conditions. The nearly identical mean delay times (horizontal lines) for evoking $I_{\text {trunc }}$ and spikes in PCs is also more consistent with a feedforward mechanism for $I_{\text {trunc }}$ because a lateral mechanism predicts an offset reflecting the sum of two synaptic delays and the activation time of interneurons. Open and filled triangles reflect PCs in layers 2 and 3 , respectively.

respectively; $p=0.31]$. The similar distributions argues against a lateral mechanism, which predicts that $I_{\text {trunc }}$ should be at a minimum $\sim 4$ ms more delayed than spikes in PCs, reflecting the two synaptic delays associated with transmission from a spiking PC to the interneuron and the interneuron to the test PC. In fact, the predicted distribution shifts for $I_{\text {trunc }}$ versus spike delays for a lateral mechanism is likely to be considerably longer, because the $4 \mathrm{~ms}$ time assumes negligible activation time for interneurons.

Are there GABAergic interneurons in the piriform cortex with properties compatible with a feedforward mechanism for $I_{\text {trunc }}$ ? To address this question, we recorded LOT stimulation-evoked responses in relatively small $(\sim 10 \mu \mathrm{m}$ diameter $)$ interneurons located at the border between layer $1 \mathrm{~b}$ and layer 2 (Fig. 4A) (see Materials and Methods). GABAergic interneurons expressing VIP or CCK are densely packed in this region, and, moreover, have axonal arborizations that extend throughout layer 2 (Ekstrand et al., 2001), making them ideal candidates for mediating the somatic $I_{\text {trunc }}$ in layer 2 PCs. In our studies, we confirmed the morphology of the putative interneurons using biocytin $(0.2 \%)$ fills and found (in three of three cells tested) that they had the dendritic characteristics reported by Ekstrand and coworkers
(2001) for VIP/CCK-expressing interneurons, including apical dendrites with short stems and sparse basal dendrites. In loose cell-attached recordings from these interneurons, we found that the LOT stimulus range that was perithreshold for eliciting spikes (failure rates, $31-64 \% ; n=6$ cells) (Fig. $4 B$ ) was quite low, similar to that seen for $I_{\text {trunc }}$ (Fig. $3 C$ ), as expected if these interneurons mediated $I_{\text {trunc }}$. Also, the delay time to spiking $($ mean $=10.0 \pm 1.8 \mathrm{~ms}$; $n=6$ cells) was similar to that observed for $I_{\text {trunc }}$ (Fig. 3D). A final property of these interneurons consistent with a feedforward mechanism for their excitation was the kinetics of the LOT stimulationevoked EPSC recorded in whole-cell voltage-clamp mode (Fig. $4 C$ ). This EPSC occurred with a short onset delay (time to half-peak, $2.0 \pm 0.1 \mathrm{~ms} ; n=4$ ), as expected if excitation of interneurons were due to direct feedforward inputs from LOT rather than a polysynaptic path involving PCs.

\section{Impact of $I_{\text {trunc }}$ on PC spiking}

We next evaluated the functional role of $I_{\text {trunc }}$ in affecting spike activity in layer 2 PCs. A starting point for this analysis was the loose cell-attached recordings from PCs (Fig. 3B), which showed that spiking, when it happened, had a very stereotypic pattern: spikes always occurred exactly once per stimulus ( $n=9$ cells) and with a delay ( $\sim 13 \mathrm{~ms}$ ) that was highly reproducible from trial to trial (SD of delay per cell, $1.5 \pm 0.2 \mathrm{~ms} ; n=9)$. We wondered whether $I_{\text {trunc }}$ might be important for affecting the number and time course of spiking in PCs, in part, because $I_{\text {trunc }}$ shared similar delay properties (Fig. 1E) and thus would be well positioned to affect spiking. Indeed, we found that blockade of $I_{\text {trunc }}$ with BMI (20 $\mu \mathrm{M})$ caused a significant increase in the number of spikes recorded in loose cell-attached recordings from PCs (from exactly one spike to $2.5 \pm 0.3$ spikes per stimulus; $n=4 ; p=0.014$ ) (Fig. $\left.5 A, B_{1}\right)$. BMI also caused PC spikes to happen with much more variable delays (SD of delay time across all spikes, $17.3 \pm 4.4 \mathrm{~ms}$; $n=4 ; p=0.039$ ) (Fig. $5 B_{2}$ ), although the degree of jitter for the first spike in BMI was unchanged (SD of delay time for first spike, $1.2 \pm 0.4 \mathrm{~ms}$ and $1.2 \pm 0.3 \mathrm{~ms}$, pre-BMI and post-BMI respectively; $n=4 ; p=0.81$ ). The BMI-induced conversion of single PC spike responses to multiple spikes dispersed across time indicates that $I_{\text {trunc }}$ has an inhibitory effect on PCs and specifically acts to maintain a precise, one-to-one relationship in the input/ output function for these cells (see Discussion). That $I_{\text {trunc }}$ limits the duration of PC excitation was also confirmed in whole-cell current-clamp recordings of subthreshold (for spiking) voltage responses in PCs (Fig. 5C). Under control conditions, the voltage response evoked by single LOT shocks (stimulus intensity $\geq 100$ $\mu \mathrm{A}$ ) included a short-duration EPSP (half-width, $6.5 \pm 1.4 \mathrm{~ms}$; $n=5$ ) followed by a long-lasting IPSP (half-width, $91 \pm 22 \mathrm{~ms}$; 
A

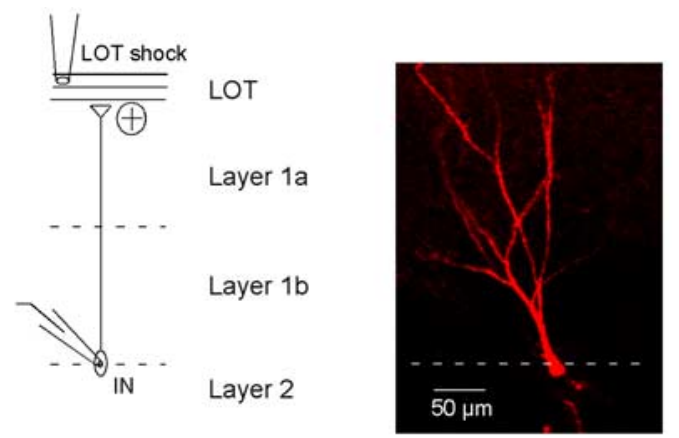

B

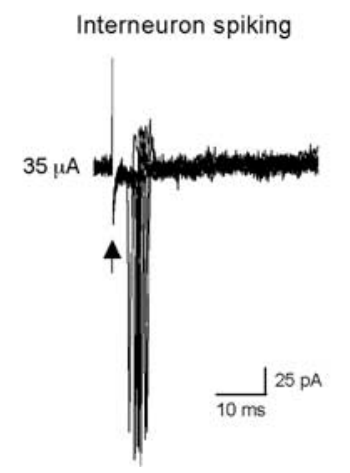

C Averaged EPSC

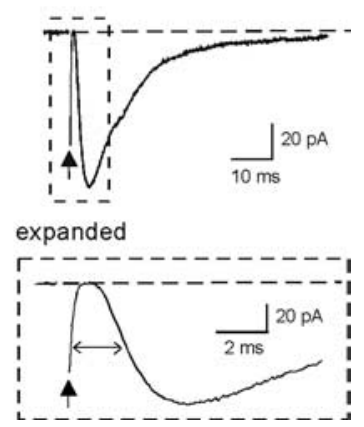

Figure 4. A subset of interneurons have properties consistent with a feedforward mechanism for $I_{\text {trunc }}$ A, Left, Diagram illustrating an interneuron (IN) juxtaposed against the different layers of the piriform cortex. All recordings were done on interneurons located at the border of layers $1 \mathrm{~b}$ and 2 , a biocytin-filled example of which is shown on the right. $\boldsymbol{B}$, Loose cell-attached recordings of interneuron activity. Ten superimposed traces are shown, five of which showed single spikes. Spiking occurred at relatively low stimulus intensities ( $35 \mu \mathrm{A}$; see summary of interneuron spike data in Fig. $3(D, D)$, as required if the interneuron is responsible for $I_{\text {trunc }}$ in $P(S$. C, EPSC evoked by LOT stimulation in the interneuron shown in $\boldsymbol{A}$ (average of 20 trials; stimulus intensity, $40 \mu \mathrm{A}$ ). The EPSC (expanded at bottom) occurred with $\sim 2$ ms delay after stimulation, as expected if it arose because of direct feedforward inputs from LOT rather than a polysynaptic path involving PCs.

$n=5$ ); in BMI, the subthreshold EPSP was much more prolonged (half-width in BMI, $97 \pm 14 \mathrm{~ms} ; n=5$ ).

The short-duration EPSP after a single LOT shock (Fig. 5C) also makes predictions about PC synaptic integration in situations in which they receive multiple mitral cell inputs at different times. Specifically, $I_{\text {trunc }}$ should limit the time window over which EPSPs can summate in PCs, leading to spike generation. To test this hypothesis, we recorded spike activity in PCs after stimulation with two electrodes placed on the LOT (100-150 $\mu \mathrm{m}$ apart), while varying the time separation $\left(\Delta t_{\text {stim }}\right)$ between the two stimuli (Fig. $6 A-C$ ). In these studies, spiking was recorded in wholecell current-clamp mode, rather than in loose cell-attached recordings, because we wanted to confirm first that the two electrodes could each effectively elicit significant excitatory and GABAergic synaptic responses, thereby reducing the variability in our results. Although this strategy meant that the intracellular chloride concentration, and thus the polarity of GABAergic events, was fixed in these experiments, the loose cell-attached recordings from PCs in Figure 5 clearly indicated that $I_{\text {trunc }}$ is inhibitory in intact PCs at voltages approaching spike threshold. To evaluate the synaptic integration window of PCs, we first adjusted the stimulus intensities such that spikes were obtained in many (50-70\%) but not all responses when the two stimuli were applied simultaneously $\left(\Delta t_{\text {stim }}=0 \mathrm{~ms}\right)$. We then tested how the spike probability $\left(P_{\text {spike }}\right)$ changed as $\Delta t_{\text {stim }}$ was increased, while keeping the stimulus intensities constant. Increasing $\Delta t_{\text {stim }}$ from

A Spiking in layer 2 PC (loose cell-attached)
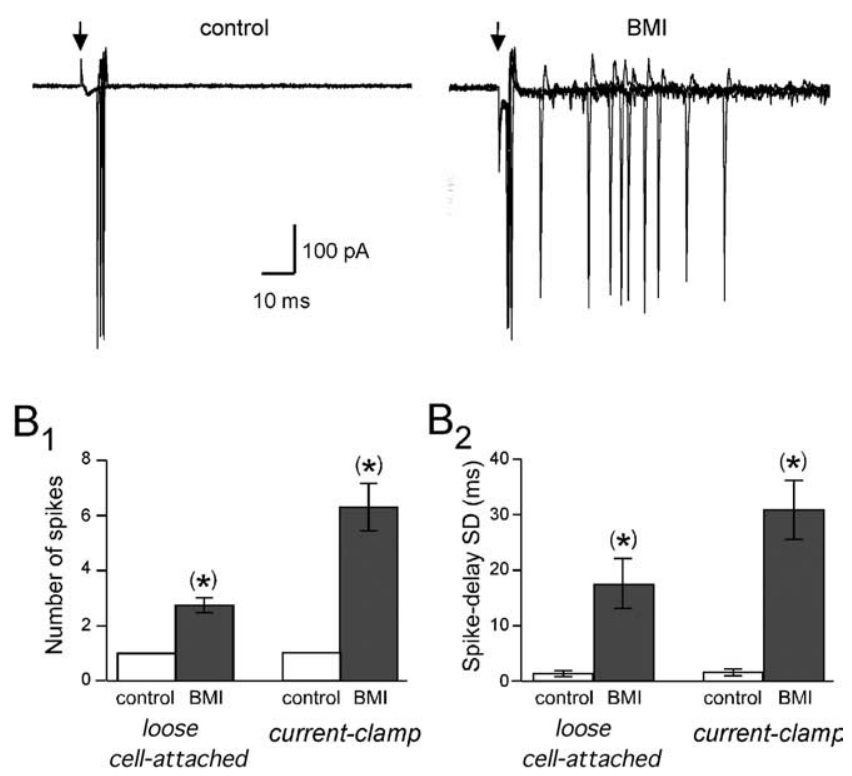

C Subthreshold voltage response in layer 2 PC
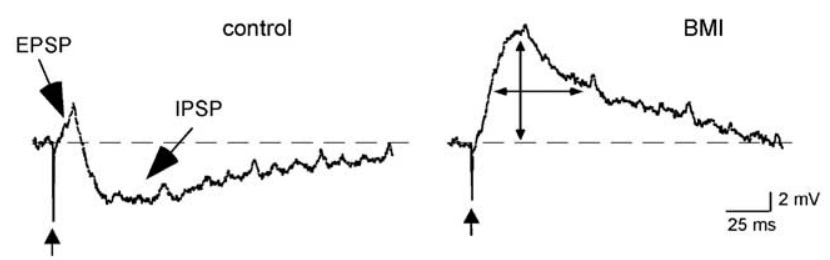

Figure 5. $I_{\text {trunc }}$ limits $\mathrm{PC}$ responses to single bouts of $\mathrm{LOT}$ input to one, precisely timed spike. $A$, Loose cell-attached recording of spiking in a layer $2 \mathrm{PC}$ in response to single LOT shocks recorded under control conditions (left) and in BMI ( $20 \mu \mathrm{M}$; right). Four superimposed trials are shown. Note the conversion from single-spike responses to multiple spikes in BMI. Spiking occurred in all four of the displayed control traces, although there was $40 \%$ failure rate across all trials in this experiment; there were no failures in BMI. $\boldsymbol{B}_{1}, \boldsymbol{B}_{2}$, Histograms comparing the number of $\mathrm{PC}$ spikes $\left(\boldsymbol{B}_{1}\right)$ and the SD of spike delays $\left(\boldsymbol{B}_{2}\right)$ before and after BMI. Data are shown from both loose cell-attached and whole-cell current-clamp recordings. The spike delays were measured from the stimulus artifacts in the raw traces to the peaks of all evoked spikes. There is no error bar for the control number of spikes because, when there was spiking, there was always exactly one spike. C, Subthreshold voltage-responses in a PC recorded in current-clamp (prestimulus membrane potential, $-50 \mathrm{mV}$ ). The control response to LOT stimulation ( $300 \mu \mathrm{A}$; left) included a short-duration EPSP, which was followed by an IPSP. BMI ( $20 \mu \mathrm{m}$; right) abolished the IPSP and also amplified and prolonged the EPSP. Traces reflect averages of 10 responses.

0 to $2 \mathrm{~ms}$ did not significantly affect $P_{\text {spike }}$ (normalized probability, $0.84 \pm 0.11 ; n=3 ; p=0.26$ ), but $P_{\text {spike }}$ was dramatically reduced when $\Delta t_{\text {stim }}$ was increased to $5 \mathrm{~ms}$ (normalized probability, $0.29 \pm 0.10 ; n=4 ; p=0.002)$. In these experiments, we could not completely exclude the possibility that the two electrodes stimulated overlapping populations of axon fibers, and thus the reduction in $P_{\text {spike }}$ between $\Delta t_{\text {stim }}=2$ and $5 \mathrm{~ms}$ could in principle have resulted from a presynaptic mechanism, for example a drop in presynaptic facilitation (Suzuki and Bekkers, 2006). However, in experiments in which we recorded dual stimulusevoked EPSCs in PCs (Fig. 6D), EPSCs evoked by the second stimulus were no different in size when $\Delta t_{\text {stim }}$ was raised from 2 to $5 \mathrm{~ms} \mathrm{(3} \pm 5 \%$ decrease in EPSC charge integral; $n=8 ; p=0.82)$. This result argued against a presynaptic effect being the cause of the reduced $P_{\text {spike }}$ for $\Delta t_{\text {stim }}=5 \mathrm{~ms}$. Also arguing that the reduction in $P_{\text {spike }}$ was due to a postsynaptic mechanism involving the 
A

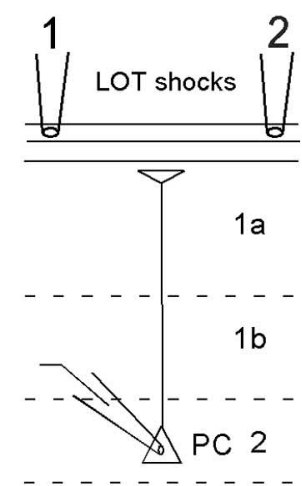

3
B

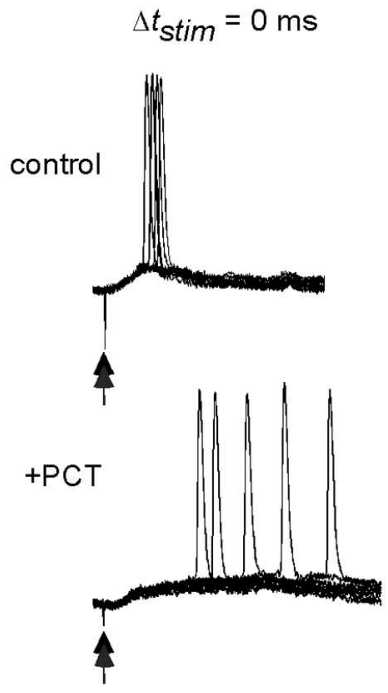

$2 \mathrm{~ms}$
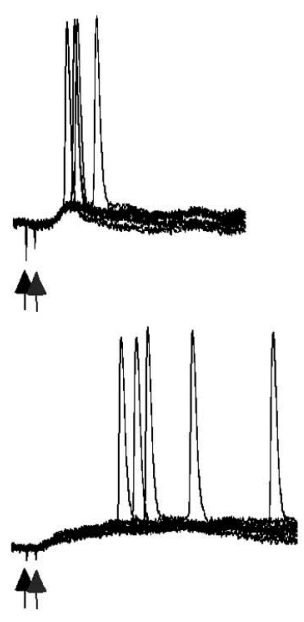

$5 \mathrm{~ms}$

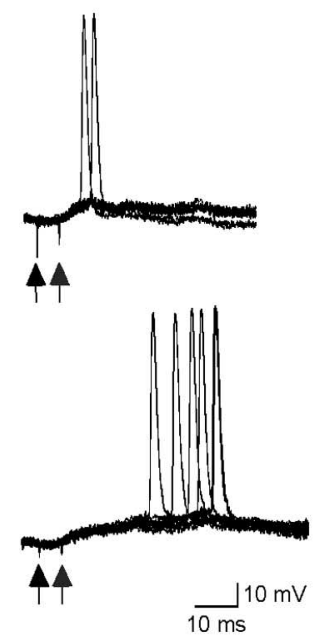

C

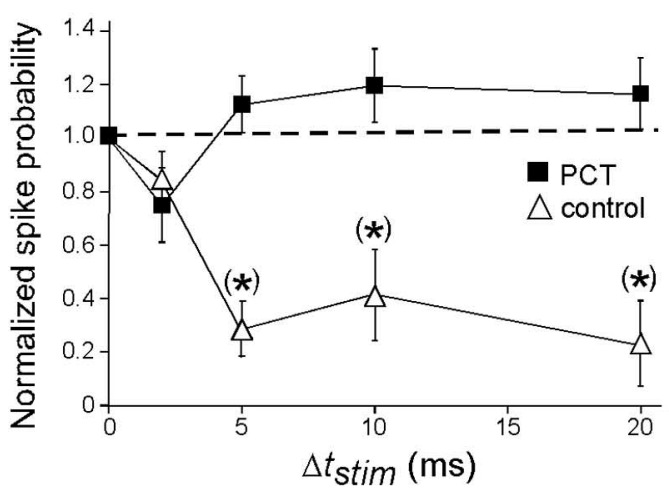

$\mathrm{D}_{1}$

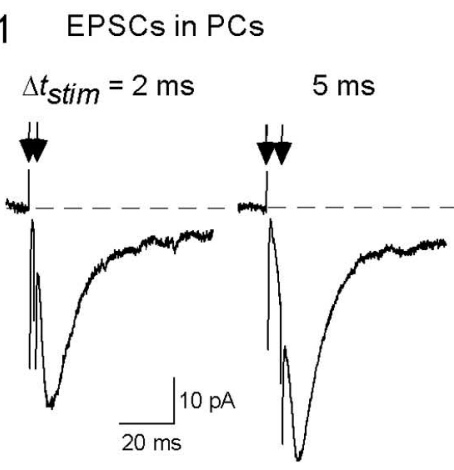

$D_{2}$

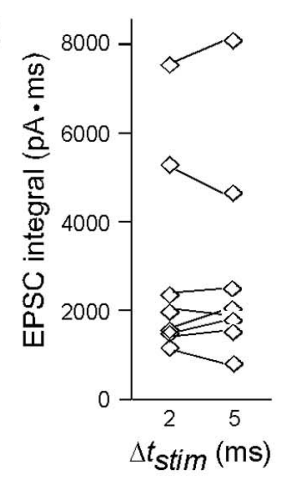

Figure 6. I Itrunc limits the synaptic integration window for PCs. A, Diagram of stimulation protocol used to evaluate the integration window. Two stimulating electrodes were positioned $100-150$ $\mu \mathrm{m}$ apart on the $L 0 T$, with stimuli being applied at various intervals $\Delta t_{\text {stim }}$. $\boldsymbol{B}$, Traces showing $\mathrm{PC}$ responses for three different $\Delta t_{\text {stim }}$ values $(0,2$, and $5 \mathrm{~ms})$ under control conditions (top) and when GABAergic activity was blocked by $100 \mu \mathrm{m}$ PCT (bottom). Each panel shows 10 superimposed traces in response to two LOT stimuli (indicated by vertical arrows). Control responses showed a significant drop in spike probability between $\Delta t_{\text {stim }}=2 \mathrm{~ms}$ ( 6 of 10 trials) and $\Delta t_{\text {stim }}=5 \mathrm{~ms}$ ( 2 of 10 trials), but, in PCT, there was no such drop. Different stimulus intensities were used in these experiments for control conditions ( $100 \mu \mathrm{A}$ for both electrodes) versus in PCT ( $50 \mu \mathrm{A}$ for both electrodes) to maintain moderate spike probabilities for $\Delta t_{\text {stim }}=0 \mathrm{~ms}$. C, Graph showing the spike probability (normalized to $\Delta t_{\text {stim }}=0 \mathrm{~ms}$ ) for $\Delta t_{\text {stim }}=0,2,5,10,15$, and $20 \mathrm{~ms}$ under control conditions and in PCT. Note, again, that the drop in spike probability compared with $\Delta t_{\text {stim }}=0 \mathrm{~ms}$ under control conditions happens mainly between $\Delta t_{\text {stim }}=2$ and $5 \mathrm{~ms}$. $D_{1}$, EPSCs in a PC evoked by the dual-electrode LOT stimulation protocol (intensity $=100 \mu \mathrm{A}$ ) for $\Delta t_{\text {stim }}=2 \mathrm{~ms}$ (left) and $\Delta t_{\text {stim }}=5 \mathrm{~ms}$ (right). Recordings were done while holding the cell at the chloride equilibrium potential $\left(-107 \mathrm{mV}\right.$ ) to minimize the $\mathrm{GABA}_{\mathrm{A}}$ receptor-mediated component of the response. Traces reflect averages of 10 responses. $D_{2}$, Summary of EPSC integral values for $\Delta t_{\text {stim }}=2 \mathrm{~ms}$ and $\Delta t_{\text {stim }}=5 \mathrm{~ms}$ for eight cells. No significant difference was seen, indicating that the sharp reduction in the control spike probability between $\Delta t_{\text {stim }}=2$ and $5 \mathrm{~ms}$ in $C$ was not a presynaptic effect. For the charge measurements, current integration began just after the second stimulus artifact in the raw data traces.

inhibitory local circuitry, we found that the $\mathrm{GABA}_{\mathrm{A}}$ receptorblocker picrotoxin (PCT) $(100 \mu \mathrm{M})$ abolished the reduction in $P_{\text {spike }}$ with increasing $\Delta t_{\text {stim }}$ (Fig. 6C). These results indicate that $I_{\text {trunc }}$ functions to limit the time window of synaptic integration in PC cells such that inputs from mitral cells arriving within $5 \mathrm{~ms}$ will be strongly favored.

\section{Discussion}

A prominent feature of odor-evoked responses in the olfactory bulb is gamma frequency $(30-70 \mathrm{~Hz})$ synchronized oscillations in the activity of the output mitral cells. The main design of our studies was to examine whether there were specific mechanisms operating at the major downstream olfactory processing center, the piriform cortex, that could be important for detecting such mitral cell input patterns.

\section{Circuit mechanisms in the piriform cortex}

We found that integration of mitral cell inputs by PCs was most profoundly affected by a population of $\mathrm{GABA}_{\mathrm{A}}$ receptormediated synaptic events that happened $\sim 10 \mathrm{~ms}$ after electrical stimulation of the LOT. These synaptic events, which we called $I_{\text {trunc }}$ based on their tendency to truncate the excitatory response in PCs generated by LOT stimulation, had the typical fast rise and decay times seen for $\mathrm{GABA}_{\mathrm{A}}$ receptor-mediated events in many other circuits (see, e.g., Kraushaar and Jonas, 2000). In functional studies, $I_{\text {trunc }}$ inhibited PC spiking in two distinct ways. First, after single LOT shocks, $I_{\text {trunc }}$ limited PC responses to single spikes that happened at nearly the same time during different trials. A tendency for GABAergic activity to produce one spike per synaptic response in PCs was shown previously in intracellular recordings done in slices by Tseng and Haberly (1988). Second, in dual LOT shock experiments, $I_{\text {trunc }}$ limited the time during which 
EPSPs could summate and cause spiking to a window of $<5 \mathrm{~ms}$. Thus, $I_{\text {trunc }}$ appears to have a general function in generating precision in the piriform cortex circuitry, both at the level of the input-output relationship of PCs and also in the requirements for incoming mitral cell inputs onto PCs.

$I_{\text {trunc }}$ had a number of specific properties that made it effective in generating precision in the piriform cortex circuitry. One was its location on PCs. $I_{\text {trunc }}$ appeared to originate at or near the PC soma, which presumably would allow it to have a more potent effect on spike initiation. An additional important feature was the onset time of $I_{\text {trunc }}$. After single LOT shocks, $I_{\text {trunc }}$ occurred relatively rapidly, in $\sim 10 \mathrm{~ms}$, and with delays that were quite invariant between trials, regardless of the strength of inhibitory network activation. We also obtained evidence that the GABAergic interneurons that drive $I_{\text {trunc }}$ are activated through a feedforward mechanism, which could explain these kinetic features. A feedforward mechanism, which is di-synaptic, should inherently be faster and less variable than more complex mechanisms such as feedback or lateral inhibition that involve additional synaptic steps. Our evidence for a feedforward mechanism for $I_{\text {trunc }}$ was mainly indirect, being based on a comparison between the $I_{\text {trunc }}{ }^{-}$ and spike-response profiles in PCs, as well as spiking in selected GABAergic interneurons. However, we did find that interneurons had short-onset EPSCs evoked by LOT stimulation, which provided direct evidence that interneurons receive feedforward inputs from mitral cells. A feedforward mechanism also fits well with the fact that the somewhat higher input resistance of GABAergic interneurons compared with PCs (see Protopapas and Bower, 2000; Suzuki and Bekkers, 2006; Zhang et al., 2006) should allow interneurons to be activated at relatively low levels of LOT inputs. In addition, there is growing evidence from studies in other brain regions that feedforward inhibition has a general circuit function in generating precise postsynaptic integration properties, in ways similar to what we observed in the piriform cortex (Pouille and Scanziani, 2001; Perez-Orive et al., 2002; Berger and Luscher, 2003; Swadlow, 2003; Mittman et al., 2005).

One aspect of the piriform cortex circuitry that we did not extensively study was recurrent excitation that arises through activation of associational inputs (Neville and Haberly, 2004). In our experiments, recurrent excitation was not clearly evident unless $\mathrm{GABA}_{\mathrm{A}}$ receptor-mediated inhibition was blocked (Fig. $1 D$, inset) $(n=3)$. Excitation at associational inputs onto PCs may however become important for PC synaptic integration under other conditions, for example, after long-term potentiation of these synapses (Kanter and Haberly, 1990; Quinlan et al., 2004). In that situation, recurrent excitation could amplify PC responses to mitral cell excitatory inputs, as long as the two types of excitation are closely matched in time.

\section{Significance for odor coding}

From the point of view of olfactory information processing, each of the two main functional effects that $I_{\text {trunc }}$ had on PC spiking may be important in specific ways. The effect of $I_{\text {trunc }}$ in limiting the $\mathrm{PC}$ response to a single spike may be critical for matching the PC to mitral cell inputs arriving as a high frequency gamma frequency pattern (Kashiwadani et al., 1999; Schoppa, 2006), in which input bouts may be separated by only 15-30 ms. The single-spike response of PCs, happening in $\sim 10 \mathrm{~ms}$, would enable PCs to respond to each round of input with exactly one spike closely locked to the input without the contaminating influence of additional spikes. Such a mechanism may be particularly important for maintaining any temporal coding feature present in the gamma oscillations (Laurent et al., 2001; Schaefer et al., 2006). The additional effect of $I_{\text {trunc }}$ in causing invariant spike delay times for different trials may have its own specific importance, because this feature could preserve information about the order in which inputs arrive onto PCs.

The effect of $I_{\text {trunc }}$ in narrowing the synaptic integration window of PCs could also affect odor coding, in this case, by making the piriform cortex favor odor-evoked synchronized signals from mitral cells (Kashiwadani et al., 1999). Indeed, a comparison between the time windows over which synchrony occurs in mitral cells (Schoppa, 2006) and synaptic integration happens in PCs, both $<5 \mathrm{~ms}$, would indicate that PCs are precisely tuned to synchronized mitral cells. Such close matching argues strongly that synchronization in mitral cells is not simply an artifact of the dynamics of the olfactory bulb circuitry but instead serves a specific function in olfactory processing.

What that function may be is not clear but a likely candidate involves the "binding" of olfactory information. Based on functional and anatomical studies (Mori et al., 1999; Lledo et al., 2005), it is now well established that odor is represented at the level of the olfactory epithelia by a map of activated odorant receptors (ORs). Such a map is also maintained at the level of mitral cell population activity in the olfactory bulb, with only perhaps some adjustment because of processes such as lateral inhibition (Yokoi et al., 1995). Our studies here, showing that the piriform cortex has specific inhibitory mechanisms that favor synchronized inputs from mitral cells, may point to connections between mitral cells and PCs as being one locus where information about different ORs is integrated together, leading to a single percept of an odor. Such a function for feedforward inhibition in signal integration requires that there be synchronization between specific subpopulations of mitral cells carrying information about different ORs, which has been shown to be possible (Schoppa, 2006). An integrating function for feedforward inhibition also requires that single PCs receive inputs from multiple subtypes of mitral cells, which, although likely, is an anatomical feature yet to be demonstrated.

\section{References}

Adrian ED (1950) The electrical activity of the mammalian olfactory bulb. Electroencephalogr Clin Neurophysiol 2:377-388.

Berger T, Lüscher HR (2003) Timing and precision of spike initiation in layer $\mathrm{V}$ pyramidal cells of the rat somatosensory cortex. Cereb Cortex $13: 274-281$.

Biedenbach MA, Stevens CF (1969) Synaptic organization of cat olfactory cortex as revealed by intracellular recording. J Neurophysiol 32:204-214.

Ekstrand JJ, Domroese ME, Feig SL, Illig KR, Haberly LB (2001) Immunocytochemical analysis of basket cells in rat piriform cortex. J Comp Neurol 434:308-328.

Franks KM, Isaacson JS (2006) Strong single-fiber sensory inputs to olfactory cortex: implications for olfactory coding. Neuron 49:357-363.

Freeman WJ (1972) Measurement of oscillatory responses to electrical stimulation in olfactory bulb of cat. J Neurophysiol 35:762-779.

Kanter ED, Haberly LB (1990) NMDA-dependent induction of long-term potentiation in afferent and association fiber systems of piriform cortex in vitro. Brain Res 525:175-179.

Kapur A, Pearce RA, Lytton WW, Haberly LB (1997) GABA $_{A}$-mediated IPSCs in the piriform cortex have fast and slow components with different properties and locations on pyramidal cells. J Neurophysiol 78:2531-2545.

Kashiwadani H, Sasaki YF, Uchida N, Mori K (1999) Synchronized oscillatory discharges of mitral/tufted cells with different molecular receptive ranges in the rabbit olfactory bulb. J Neurophysiol 82:1786-1792.

Ketchum KL, Haberly LB (1993) Membrane currents evoked by afferent fiber stimulation in rat piriform cortex. I. Current source-density analysis. J Neurophysiol 69:248-260.

Kraushaar U, Jonas P (2000) Efficacy and stability of quantal GABA release 
at a hippocampal interneuron-principal neuron synapse. J Neurosci 20:5594-5607.

Laurent G, Stopfer M, Friedrich RW, Rabinovich MI, Volkovskii A, Abarbanel HD (2001) Odor encoding as an active, dynamical process: experiments, computation, and theory. Annu Rev Neurosci 24:263-297.

Lledo PM, Gheusi G, Vincent JD (2005) Information processing in the mammalian olfactory system. Physiol Rev 85:281-317.

Miles R, Tóth K, Gulyás AI, Hájos N, Freund TF (1996) Differences between somatic and dendritic inhibition in the hippocampus. Neuron 16:815-823.

Mittman W, Koch U, Hausser M (2005) Feed-forward inhibition shapes the spike output of cerebellar Purkinje cells. J Physiol 563:369-378.

Mori K, Nagao H, Yoshihara Y (1999) The olfactory bulb: coding and processing of odor molecule information. Science 286:711-715.

Neville KR, Haberly LB (2004) Olfactory cortex. In: The synaptic organization of the brain, Ed 5 (Shepherd GM, ed) pp 415-454. New York: Oxford UP.

Perez-Orive J, Mazor O, Turner GC, Cassenaer S, Wilson RI, Laurent G (2002) Oscillations and sparsening of odor representations in the mushroom body. Science 297:359-365.

Pouille F, Scanziani M (2001) Enforcement of temporal fidelity in pyramidal cells by somatic feed-forward inhibition. Science 293:1159-1163.

Protopapas AD, Bower JM (2000) Physiological characterization of layer III non-pyramidal neurons in piriform (olfactory) cortex of rat. Brain Res 865:1-11.

Quinlan EM, Lebel D, Brosh I, Barkai E (2004) A molecular mechanism for stabilization of learning-induced synaptic modifications. Neuron 41:185-192.
Rodriguez R, Haberly LB (1989) Analysis of synaptic events in the opossum piriform cortex with improved current source-density techniques. J Neurophysiol 61:702-718.

Schaefer AT, Angelo K, Spors H, Margrie TW (2006) Neuronal oscillations enhance stimulus discrimination by ensuring action potential precision. PLoS Biol 4:e163.

Schoppa NE (2006) Synchronization of olfactory bulb mitral cells by precisely timed inhibitory inputs. Neuron 49:271-283.

Schoppa NE, Kinzie JM, Sahara Y, Segerson TP, Westbrook GL (1998) Dendrodendritic inhibition in the olfactory bulb is driven by NMDA receptors. J Neurosci 18:6790-6802.

Stopfer M, Bhagavan S, Smith BH, Laurent G (1997) Impaired odour discrimination on desynchronization of odour-encoding neural assemblies. Nature 390:70-74.

Suzuki N, Bekkers JM (2006) Neural coding by two classes of principal cells in the mouse piriform cortex. J Neurosci 26:11938-11947.

Swadlow HA (2003) Fast-spike interneurons and feedforward inhibition in awake sensory neocortex. Cereb Cortex 13:25-32.

Tseng GF, Haberly LB (1988) Characterization of synaptically mediated fast and slow inhibitory processes in piriform cortex in an in vitro slice preparation. J Neurophysiol 59:1352-1376.

Yokoi M, Mori K, Nakanishi S (1995) Refinement of odor molecule tuning by dendrodendritic synaptic inhibition in the olfactory bulb. Proc Natl Acad Sci USA 92:3371-3375.

Zhang C, Szabó G, Erdélyi F, Rose JD, Sun QQ (2006) Novel interneuronal network in the mouse posterior piriform cortex. J Comp Neurol 499: $1000-1015$. 